\title{
Comparative Study of Uric Acid Levels between Rural and Urban Populations
}

\author{
M. Jansi Rani Sivasubramanian¹, M. Kajalakshmy², Hemavathi Baskar³, \\ Abirami Soundararajan ${ }^{4}$, Rhutesh Mohavanam ${ }^{5}$, Thanmayaananth ${ }^{6}$
}

\begin{abstract}
${ }^{1}$ Department of Biochemistry, Sri Lakshmi Narayana Institute of Medical Sciences, Puducherry, India. ${ }^{2}$ Department of Biochemistry, Sri Lakshmi Narayana Institute of Medical Sciences, Puducherry, India. ${ }^{3}$ Department of Physiology, Government Vellore Medical College, Tamilnadu, India. ${ }^{4}$ Department of Biochemistry, Government Hospital, Oothangarai, Tamilnadu, India. ${ }^{5}$ Department of Dentistry, Venkateshwara Medical College, Chennai, Tamilnadu, India. ${ }^{6}$ PIMS, Puducherry, India.
\end{abstract}

\section{ABSTRACT}

\section{BACKGROUND}

The most common form of inflammatory arthritis is gout and is typically characterized by hyperuricemia and monosodium urate crystal deposition predominantly in the joints and the urinary tract. High plasma uric acid (UA) is a precipitating factor for gout, renal calculi, metabolic syndrome and cardiovascular disease. The main causes for higher plasma UA are higher synthesis, lower excretion or both. The triglycerides synthesis in the liver is associated with the de novo synthesis of purine, increasing uric acid production. The role played by diet on hyperuricemia has not yet been fully understood, but high intake of alcohol mainly beer and fructose-rich industrialized food seem to influence uric acid production. In developed countries, over the past two decades, the prevalence of gout and hyperuricemia has increased, and research has become progressively more actively carried out in those areas. Although $>600$ related clinical studies have been published to date regarding gout and hyperuricemia, the quality of care for gout and hyperuricemia remains suboptimal. Hyperuricemia is a risk factor for many diseases that has been poorly researched, in and around Pondicherry in both rural and urban population and its prevalence is largely unknown.

\section{METHODS}

This is a cross sectional survey involving 100 subjects. $0.5 \mathrm{~mL}$ of random blood sample was collected, and serum uric acid was determined by auto analyser method.

\section{RESULTS}

The uric acid level in rural population area was higher by $9 \%$ when compared to urban population. The mean serum uric acid was higher in rural population. (9.32 \pm 1.073$)$.

\section{CONCLUSIONS}

The prevalence of hyperuricemia is high in rural area in this study. There is a need for more research considering implications. Long term follow-up is needed. Proper control of uric acid to less than $6 \mathrm{mg} \%$ is the main aim to prevent comorbidities. Patient education is very important.

\section{KEY WORDS}

Uric Acid, Hyperuricemia, Fructose, Chronic Kidney Disease, Urban Population, Comorbidities, Gout
Corresponding Author: M. Jansi Rani Sivasubramanian, \#19, $7^{\text {th }}$ Cross, Kambanagar, Reddiyarpalayam, Puducherry-10, India.

E-mail: onenesjrs292@gmail.com

DOI: 10.14260/jemds/2020/187

Financial or Other Competing Interests: Dr. Sivasubramanian Reports Grants from BIHER Deemed University, During the Conduct of the Study.

How to Cite This Article: Sivasubramanian MJR, Kajalakshmy $M$, Baskar H, et al. Comparative study of uric acid levels between rural and urban populations. J. Evolution Med. Dent. Sci. 2020;9(11):869-874, DOI:
Submission 03-12-2019,

Peer Review 22-02-2020,

Acceptance 29-02-2020,

Published 16-03-2020. 


\section{BACKGROUND}

Uric acid is the final catabolic product of purine or nucleotide metabolism. About two-thirds of uric acid is excreted by glomerular filtration. ${ }^{[1,2]}$ Purine nucleotides are obtained from both endogenous sources like de novo molecule synthesis and nucleic acid break down and exogenous sources mainly alimentary intake. De novo purines synthesis depends on the 5-phosphoribosyl-l-pyrophosphate compound, which is converted to inosinic acid enzymatically. It may either be converted into bases for inclusion into nucleic acids or broken down into xanthine to form uric acid. Synthesis of Purine nucleotide also occur through the activities of two different enzymes, catalysing the single-step synthesis of a purine nucleotide from a purine base substrate. During the reverse process hypoxanthine, the intermediate breakdown product can be salvaged by the enzyme hypoxanthine-guanine phosphoribosyl transferase and then re-incorporated into nucleus. This pathway is regulated and controlled by feedback inhibition. Uric acid is a weak acid, with ionization constant of acid (pKa) of 5.75-10.3. At 7.4 physiological $\mathrm{pH}$, in the extracellular compartment, $99 \%$ of uric acid is in the ionized form as urate (as monosodium urate in blood and as ammonium potassium and calcium urate in urine). When $\mathrm{pH}$ falls to 5.7, in the urinary tract, uric acid formation is favoured. Physiological daily amount of exogenous and endogenous uric acid production is about $700 \mathrm{mg}$, which is balanced by an equal output via urine and feces. As previously mentioned, $30 \%$ of uric acid is broken down by intestinal flora and excreted through the stool, while the remaining 70\% (approximately $500 \mathrm{mg}$ per day) is excreted unchanged form through the kidneys. As a consequence, under normal conditions uric acid production and excretion are carefully balanced.

Hyperuricemia has been associated with high risk of peripheral and coronary artery disease, cardiovascular disease, hypertension, diabetes mellitus and CKD.[3] Asymptomatic hyper uricemia progress into episodes of gout acute attacks with asymptomatic intervals, chronic gouty arthritis and destruction of joints. ${ }^{[4]}$ Gout is a condition characterized by deposition of uric acid crystals in various joints.[5]. Prevalence of gout is up to $4 \%$ in western countries. Gout is one of the most common inflammatory neuropathies. Hyperuricemia leads to crystal formation, aggregation and deposit as monosodium urate (MSU) crystals in joints and soft tissues. Acute inflammatory reaction is induced by this crystals. There will be massive leukocyte recruitment and local release of chemokines, cytokines, proteolytic enzymes and reactive oxygen species. Once established gout present with features of excruciating pain, redness, swelling in joints and several cardiovascular and kidney related comorbidities. Recently gout has gained renewed interest which has led to improvement in its management. Number of publications has doubled from 2009 to 2017. Physiological mechanism behind hyperuricemia is increased production of urate from diet, reduced renal or gut excretion of uric acid and or endogenous substrate that raise purine. ${ }^{[6]}$

\section{Genetics}

Serum uric acid level is influenced by combination of environmental exposure and inherited genetic variants. Several genome wide association studies (GWAS) in past discovered the genome for urate specific loci.[7] The proteins (SLC family gene) involved in urate transportation dominate these loci. Recently genetic loci influencing serum uric acid were identified. Those with strongest influence include ABCG2, NPT4 (SLC17A3), OAT4 (SLC22A11), NPT1 (SLC17A1), URAT1 (SLC2212), GLUT9 (SLC2A9). In addition to this above strong genetic factors, dietary factors also act independently or interactively with individual's genetic risk for hyperuricemia. ${ }^{[8]}$

\section{Epigenetics in Gout}

DNA methylation altered patterns, micro RNA (miRNA) implied as a causative factor in pathogenesis of gout. Study conducted by Zhou et al[9] analyses the expression profiles of mIRNA in causing gout. They observed miR-920 and miR-488 are decreased in gout patients. Their findings proposed miRNA as a regulator in gout development.

\section{Pathogenesis}

Serum uric acid elevation is the main pathogenesis of gout which leads to the formation of mono sodium urate crystals (MSU).Once crystals are formed they are deposited in joints initiating inflammatory cascade path way causing acute gout. The key risk factor for gout is hyperuricemia. Although all patients with hyperuricemia does not develop gout. Despite monosodium urate crystal deposition, gout patients have intermittent flares. Recently more attention has been paid in finding out the mechanism of development of gout. Dong et al[10] found 2 trans membrane transporter activity related pathways in regulating uric acid levels associated with gender and body mass index influence. They also found indirect role of ATP-P2X7R signalling pathway in gout mechanism. Metabolic components also play additional role to elicit inflammation. The combination of all these indicate continuous development of hyperuricemia into gout. Recent research show that consumption of sea foods is positively linked with hyperuricemia[11] and fructose containing soft drinks ${ }^{[12]}$ and food as well as legume intake in animal studies. Incidence of gout in India is not clear.

Hyperuricemia is a condition characterized by elevated uric acid level in the blood. The normal upper limit is 6.8 $\mathrm{mg} / \mathrm{dl}$, and anything above $7 \mathrm{mg} / \mathrm{dl}$ is considered saturated, and clinical feature scan occur. It is estimated that $21 \%$ of general population and $25 \%$ of hospitalized patients have asymptomatic hyperuricemia. An Indian study revealed that urban people is involved more common than rural people and due to increased prevalence of metabolic syndrome. Since there is limited Indian study, the present study aimed to compare the uric acid levels between urban and rural population and find the pathogenesis.

\section{METHODS}

The study was conducted at department of Biochemistry SLIMS, Pondicherry during the period from September 2017 to October 2018. A cross sectional method was included in this study. Sample size is fixed based on test kit and self-fund. Both male and female patients of total 200 (urban -100 , rural-100) with 40-50 years of age, serum uric acid $>6 \mathrm{mg} / \mathrm{dl}$ are included 
in this study. Those who are not under treatment with uric acid lowering drugs, arthritis, gout attack in last 2 weeks, peptic ulcer, serious haematological disorder, uncontrolled DM, who require dialysis, being treated with systemic immunosuppressant were excluded from the study. The study was approved by IEC and informed consent was obtained from all participants.

\section{Estimation}

$0.5 \mathrm{~mL}$ of random blood sample was collected, and serum uric acid was determined by auto analyser method.

\section{Statistical Analysis}

SPSS version 16.0 was used for statistical analysis. The value were represented in mean, median and standard deviation. The statistical test used were unpaired or independent sample $\mathrm{t}$ test for comparison of mean value of 2 groups. P value $<0.05$ was considered statistically significant.

\section{RESULTS}

\begin{tabular}{|c|c|c|c|c|}
\hline & Mean & Standard Deviation & T Test & p Value* \\
\hline Rural & 9.32 & 1.073 & \multirow{2}{*}{9.996} & 0.000 \\
\hline Urban & 8.1 & 0.583 & & \\
\hline \multicolumn{3}{|c|}{ Table 1. Comparative Analysis of Serum uric Acid between } \\
Rural and Urban Population Using Student t Test \\
\hline
\end{tabular}

The mean serum uric acid level was higher in rural population when compared to urban population and it was statistically significant. The mean value by using unpaired or independent sample $t$ test mean value comparison was done.

\section{DISCUSSION}

In this study mean serum uric acid levels and prevalence of hyperuricemia were found to be higher in rural than urban population. The main pathogenesis behind hyperuricemia is under excretion of uric acid, over production of uric acid. The serum uric acid level depends on dietary purines, endogenous purines degradation, and the renal and intestinal excretion of urate. The main factor contributing to hyperuricaemia is under-excretion of urate. High ingestion of purine sources mainly animal protein meat, seafood, beer[13] and alcohol increases uric acid production. Alcohol intake increases uric acid concentrations by reducing excretion ${ }^{[14,15]}$ and increasing urate production.[16] Fructose high intake increases serum uric acid, a relationship that has been ascribed to fructose phosphorylation in the liver with subsequent ATP regeneration and depletion.

Meta-analysis has recently shown that elevation in uric acid level related to an increase in coronary artery disease infarction and mortality events, ${ }^{[17]}$ and uric acid is an independent risk factor for cardiovascular diseases ${ }^{[18],[19]}$ particularly in diabetic and hypertensive patients ${ }^{[20]}$ shown by epidemiological studies. It is also speculated that uric acid is one of the risk factor for metabolic syndrome. ${ }^{[21]}$ High uric acid levels in individuals have an odds ratio of 1.6-fold higher for developing MS.[22]
Higher uric acid levels are associated with Metabolic Syndrome ${ }^{23]}$ and its components,[24] obesity, dyslipidemia, hypertension,[25] insulin resistance, increased C-reactive protein concentration, [26] endothelial dysfunction ${ }^{[27]}$ and cardiovascular diseases risk.[28],[29],[30] Asymptomatic hyperuricemia progress into acute gout attack episodes with asymptomatic intervals, chronic gouty arthritis and destruction of joints. In $85 \%$ to $90 \%$ of people hyper uricemia is the major contributor to gout develops due to under excretion of urate (excretion $<330 \mathrm{mg} / \mathrm{dl}$ ). In $10-15 \%$ of hyperuricemia cases over production (excretion $>600 \mathrm{mg} / \mathrm{dl}$ ) accounts. The common precipitants of hyperuricemia include strenuous exercise, cold, alcoholism, over eating, ethanol ingestion, accelerate degradation of ATP into AMP (precursor of uric acid)

\section{Experimental Studies}

The detrimental effect of some diet on hyperuricaemic risk and gout progression has been focused by several clinical studies. Recent data evaluated the diet, genetic risk score and sex association on uric acid levels. It was found that gene, diet interactions found to influence serum uric acid levels. The main dietary factors which found to influence serum uric acid level was legumes in general population, Vit $\mathrm{C}$ among men, red meat among women. Experimental evidence proved that antihyperuricaemic and anti-inflammatory effects of plant derived components in gout induced inflammation. Polyphenols and fibres have been studied last decade. Those studies proved that SCFAs from metabolism of fibres are able to inhibit the inflammation in MSU crystals in mice. In this study mice were given high fibre diet of 2 weeks before injecting MSU crystals in mice. ${ }^{[31]}$ After high fibre diet inflammation has been reduced faster when compared to low fibre diet. The polyphenol beneficial effects has been recently revived. These plant derived compounds modulate the multiple inflammatory pathways. In gout polyphenol reduces the serum uric acid by decreasing Xanthine oxidase activity and by inhibiting NF-B transcriptional factor and activating

\section{Diet and Lifestyle Changes}

The role played by the diet in gout has received strong attention over the last decade. From the evidence it is clear that diet is partially responsible for fluctuation of serum uric acid levels in blood. Hyperuricaemic patients are recommended to reduce food causing hyperuricemia such as high purine rich diets, sweetened and alcoholic beverages. EULAR 2016 guidelines mentioned that individuals with gout should receive advice regarding diet and lifestyle changes. Hyperuricemia has shown to associated with high blood pressure and hypertension. Following DASH diet (dietary approaches to stop hypertension) help in decreasing uric acid levels in serum. This diet helps in intake of fruits, vegetables, low fat products, whole grains, limiting sugars, sweetened beverages and saturated fats. Following DASH diet has been associated with reduced intake of gout in men. The effect has been initiated within 30 days of diet initiation and maintained at 90 days.

\section{Physical Activity}

With regard to gout prevention, physical activity is ignored. Cross sectional studies have shown that Serum uric acid is 
declined weekly in long distance running and uric acid is lowered in athletes.[32]

Uric acid level can be lowered by following ideal body weight achievement, smoking cessation, optimal exercise and health and maintenance of good hydration. Purine rich foods (organ meat-liver and kidney, sea food, thymus and pancreas of calf and lamb), fructose rich drinks (sweetened soft drinks, corn syrup, ice cream) and fruits (oranges and apples), alcohol use especially during the attacks of gout and large rich meals should be avoided. Among food components, fructose affects uric acid and has been associated with higher risk of developing gout. High fructose ingestion causes consumption and degradation of adenosine nucleoside (ATP/AMP hydrolysis) resulting in accumulation of uric acid in circulation. A nationwide population based cohort study regarding alcohol consumption recently revealed that strong association between alcohol dependence disease, alcohol related disease and gout. By community survey in people with gout alcohol is the most common trigger of gout. Clinical evidence proving that elevated uric acid is associated strongly with abnormal glucose metabolism, insulin resistance, high blood pressure and prevalence of hypertension. A recent studies reviewed the weight loss effects in over weight out patients by measuring uric acid levels. Moderate evidence suggest that at short or long term follow up weight loss have beneficial effects on serum uric acid levels.

\section{Limit}

Beef, lamb, pork and sea food (shell fish and sardines), natural sugars, sweetened beverages, desserts and table salts. Limit alcohol especially beer, also wine and spirits. Low fat, nondairy products and vegetables intake should be encouraged. Vitamin C and coffee has been associated with decreased serum uric acid levels and thereby reduce risk factors for comorbidities. Zhang et al concluded that low to moderate education was considered as risk factor for hyperuricemia. Some researchers found that well educated people appear to eat balanced, healthier diet. People who are having low education have less knowledge regarding nutrition and pay less attention to diet.[33]

In order to control gout and prevent damage to joints early diagnosis is important. Many techniques are available to check the monosodium urate crystals. However techniques are most expensive and non-available. So, clinicians have to rely on patient history and clinical features. The gold standard to measure gout is identification of monosodium urate crystals (MSU) in synovial fluid (SF).This test has specificity of $100 \%$. The immediate diagnosis is done by assessing the needle like shaped crystals with strong negative birefringence in undiagnosed patients of gout and in patients with established joint disease. The professionals has done recent study on synovial fluid analysis by examining crystals. They mentioned that MSU crystals are most recognizable crystals. Experiments carried out regarding monosodium urate crystals reported that unlike calcium pyrophosphate crystals (CPP), MSU are well recognized even without centrifugation of synovial fluid. Due to small joint synovial fluid aspiration and invasiveness the replacement procedure is researched for gout diagnosis.

$\mathrm{X}$ ray is considered as a fast method to assess joint damage. But to diagnose gout in early stages, X-ray is of little help. To assess the joint damage gout modified sharp/van ER Heidje scoring method has been established. The European league against rheumatism (EULAR) and ACR recognized the value of dual energy computer tomography (DECT) and ultrasound (US) as scoring items for classification of gout. In diagnosing crystal related arthropathies musculoskeletal ultrasound (MSUS) has demonstrated good sensitivity and specificity. The current task of outcome measures in rheumatology clinical trials (OMERACT) MSUS group is standardization and validation of MSUS crystals. This task is done to improve the usage of this technique in clinical practice.

A study was carried out in 89 enrolled arthritis patients. Ultrasound showed crystal deposition and confirmed the diagnosis of gout. Retrospectively the sensitivity and specificity of double contour sign were $42 \%$ and $92 \%$. For intra articular aggregates sensitivity and specificity were $58 \%$ and $92 \%$ and for tophi $40 \%$ and $100 \%$ [34].For diagnosing gout ultrasound has demonstrated high specificity and good accuracy. Dual -energy computer tomography (DECT) is a modified computer tomography. This technique uses two Xray beams. On the basis of relative absorption of $\mathrm{X}$ rays at different photon energy levels DECT differentiate deposits in soft tissues. Because of it cost and ionizing radiation hazards exposure to the patient is limited in clinical practice.

Recently meta-analysis and systemic literature review concluded that in diagnosing intra or extra articular tophaceous gout DECT has high diagnostic accuracy. In patients with uncertain diagnosis it can be used as second line imaging modality. DECT when compared to ultrasound underestimate the size of tophi and inflammation. It has been demonstrated that in short duration of time DECT has limited diagnostic sensitivity.

\section{Management}

Formation of monosodium urate crystal may be prevented and reversible. Uric acid level should be reduced below $6 \mathrm{mg} / \mathrm{dl}$, below the limit of solubility to reduce gout. At present allopurinol, Xanthine oxidase inhibitor first line drug and febuxostat second line drug are recommended as uric acid lowering drugs. These drugs acts by inhibiting uric acid production. Recently in clinical practice Lesinurad, an uricosuric agent has entered.[35] This drug acts by inhibition of OAT4 and URAT1 renal transporters by increasing renal uric acid excretion and by inhibiting uric acid reabsorption. When compared to other uricosuric drugs, Lesinurad exhibit decreased drug-drug interaction and side effects. Similar study was conducted in Ganzi Tibetan Autonomous Prefecture, China. Being farmers-herdsmen, low to moderate education level, hypertension, current drinking and having higher BMI) and creatinine all contributed to hyperuricemia in this population. Liu et al conducted a Cross sectional study in china to assess uric acid levels. He found that uric acid was higher in urban compared to rural population. ${ }^{[36]}$

\section{ACKNOWLEDGEMENT}

Authors thank Mrs. Tamilselvi, Statistician, Department of P\&SM, SLIMS, Pondicherry. 


\section{CONCLUSIONS}

The prevalence of hyperuricemia is high in rural area in this study. There is a need for more research considering implications. Long term follow-up is needed. Proper control of uric acid to less than $6 \mathrm{mg} \%$ is the main aim to prevent comorbidities. Patient education is very important.

\section{REFERENCES}

[1] Harrison R. Structure and function of xanthine oxidoreductase: where are we now? Free Radic Biol Med 2002;33(6):774-97.

[2] Sautin YY, Johnson RJ. Uric acid: the oxidant-antioxidant paradox. Nucleosides Nucleotides Nucleic Acids 2008;27(6):608-19.

[3] Haque T, Rahman S, Islam S, et al. Assessment of the relationship between serum uric acid and glucose levels in healthy, prediabetic and diabetic individuals. Diabetology \& Metabolic Syndrome 2019;11:49.

[4] Tyagi S, Rawat S, Saxena S. Comparative study between febuxostat and allopurinol based on safety and urate lowering efficacy in gouts patients of Indian population. Int J Curr Microbiol App Sci 2016;5(10):801-15.

[5] Ragab G, Elshahaly M, Bardin T. Gout: an old disease in new perspective - a review. J Adv Res 2017;8(5):495-511.

[6] Maiuolo J, Oppedisano F, Gratteri S, et al. Regulation of uric acid metabolism and excretion. Int J Cardiol 2016;213:8-14

[7] Major TJ, Dalbeth N, Stahl EA, et al. An update on the genetics of hyperuricaemia and gout. Nat Rev Rheumatol 2018;14(6):341-53.

[8] Karwur FF, Pujiastuti DR. Uric acid homeostasis and disturbances. Folia Medica Indonesiana 2017;53(4):2928.

[9] Zhou W, Wang Y, Wu R, et al. MicroRNA-488 and -920 regulate the production of pro-inflammatory cytokines in acute gouty arthritis. Arthritis Res Ther 2017;19:203.

[10] Dong Z, Zhou J, Xu X, et al. Genetic variants in two pathways influence serum urate levels and gout risk: a systematic pathway analysis. Sci Rep 2018;8(1):3848.

[11] Villegas R, Xiang YB, Elasy T, et al. Purine-rich foods, protein intake and the prevalence of hyperuricemia: The Shanghai Men's Health Study: Nutr Metab Cardiovasc Dis 2012;22(5):409-16.

[12] De Oliveira EP, Burini RC. High plasma uric acid concentration: causes and consequences. Diabetol Metab Syndr 2012;4:12.

[13] Yu KH, See LC, Huang YC, et al. Dietary factors associated with hyperuricemia in adults. Semin Arthritis Rheum 2008;37(4):243-50.

[14] Drum DE, Goldman PA, Jankowski CB. Elevation of serum uric acid as a clue to alcohol abuse. Arch Intern Med 1981;141(4):477-9.

[15] Eastmond CJ, Garton M, Robins S, et al. The effects of alcoholic beverages on urate metabolism in gout sufferers. Br J Rheumatol 1995;34(8):756-9.

[16] Faller J, Fox IH. Ethanol-induced hyperuricemia: evidence for increased urate production by activation of adenine nucleotide turnover. N Engl J Med 1982;307(26):1598602.

[17] Kim SY, Guevara JP, Kim KM, et al. Hyperuricemia and risk of stroke: a systematic review and meta-analysis. Arthritis Rheum 2009;61(7):885-92.

[18] Chen JH, Chuang SY, Chen HJ, et al. Serum uric acid level as an independent risk factor for all-cause, cardiovascular and ischemic stroke mortality: a Chinese cohort study. Arthritis Rheum 2009;61(2):225-32.

[19] Takahashi MM, de Oliveira EP, de Carvalho AL, et al. Metabolic Syndrome and dietary components are associated with coronary artery disease risk score in freeliving adults: a cross-sectional study. Diabetol Metab Syndr 2011;3:7.

[20] Feig DI, Johnson RJ. Hyperuricemia in childhood primary hypertension. Hypertension 2003;42(3):247-52.

[21] Chen LY, Zhu WH, Chen ZW, et al. Relationship between hyperuricemia and metabolic syndrome. J Zhejiang Univ Sci B 2007;8(8):593-8.

[22] Onat A, Uyarel H, Hergenc G, et al. Serum uric acid is a determinant of metabolic syndrome in a populationbased study. Am J Hypertens 2006;19(10):1055-62.

[23] Sui X, Church TS, Meriwether RA, et al. Uric acid and the development of metabolic syndrome in women and men. Metabolism 2008;57(6):845-52.

[24] Abdullah AR, Hasan HA, Raigangar VL. Analysis of the relationship of leptin, high-sensitivity c-reactive protein, adiponectin, insulin and uric acid to metabolic syndrome in lean, overweight, and obese young females. Metab Syndr Relat Disord 2009;7(1):17-22.

[25] Doehner W, Schoene N, Rauchhaus M, et al. Effects of xanthine oxidase inhibition with allopurinol on endothelial function and peripheral blood flow in hyperuricemic patients with chronic heart failure: results from 2 placebo-controlled studies. Circulation 2002;105(22):2619-24.

[26] Saito M, Ishimitsu T, Minami J, et al. Relations of plasma high-sensitivity C-reactive protein to traditional cardiovascular risk factors. Atherosclerosis 2003;167(1):73-9.

[27] Farquharson CA, Butler R, Hill A, et al. Allopurinol improves endothelial dysfunction in chronic heart failure. Circulation 2002;106(2):221-6.

[28] Gagliardi ACM, Miname MH, Santos RD. Uric acid: a marker of increased cardiovascular risk. Atherosclerosis 2009;202(1):11-7.

[29] Burack RC, Keller JB, Higgins MW. Cardiovascular risk factors and obesity: are baseline levels of blood pressure, glucose, cholesterol and uric acid elevated prior to weight gain? J Chronic Dis 1985;38(10):865-72.

[30] Fang J, Alderman MH. Serum uric acid and cardiovascular mortality the NHANES I epidemiologic follow-up study, 1971-1992. National Health and Nutrition Examination Survey. JAMA 2000;283(18):2404-10.

[31] Vieira AT, Galvão I, Macia LM, et al. Dietary fiber and the short-chain fatty acid acetate promote resolution of neutrophilic inflammation in a model of gout in mice. J Leukoc Biol 2017;101(1):275-84.

[32] Williams PT. Effects of diet, physical activity and performance and body weight on incident gout in ostensibly healthy, vigorously active men. American Journal of Clin Nutrition 2008;87(5):1480-7. 
[33] Zhang X, Meng Q, Feng J, et al. The prevalence of hyperuricemia and its correlates in Ganzi Tibetan Autonomous Prefecture, Sichuan Province, China. Lipids in Health and Disease 2018;17:235.

[34] Pattamapaspong N, Vuthiwong W, Kanthawang T, et al. Value of ultrasonography in the diagnosis of gout in patients presenting with acute arthritis. Skeletal Radiol 2017;46(6):759-67.
[35] Scirè CA, Rossi C, Punzi L, et al. Change gout: how to deal with this "silently-developing killer" in everyday clinical practice. Curr Med Res Opin 2018;34(8):1411-17.

[36] Liu H, Zhang XM, Wang YL, et al. Prevalence of hyperuricemia among Chinese adults: a national crosssectional survey using multistage, stratified sampling. J Nephrol 2014;27(6):653-8. 